\title{
Polyphase Sequence Design using a Genetic Algorithm
}

\author{
Hai Huyen Dam*, Hans-Jürgen Zepernick*, and Helge Lüders ${ }^{\dagger}$ \\ *Western Australian Telecommunications Research Institute \\ 39 Fairway, Nedlands, WA 6907, Australia \\ Email: \{dam, hans\}@watri.org.au \\ ${ }^{\dagger}$ Aachen University of Technology \\ Templergraben 55, 52056 Aachen, Germany \\ Email: helge@fueg.de
}

\begin{abstract}
The performance of direct sequence code division multiple access (DS-CDMA) systems depends on the autocorrelation and cross-correlation properties of the deployed spreading sequences. Since good auto-correlation characteristics come at the expense of the cross-correlation properties and vice versa, a combination of these two measures needs to be optimized when designing a DS-CDMA system. In this paper, we consider the design of complex-valued spreading sequences with optimized correlation characteristics. In particular, the maximum nontrivial aperiodic correlation values are used to specify the cost or fitness function for the optimization problems. A genetic algorithm is presented for the design of polyphase sequences, namely, Oppermann sequences and modified Walsh-Hadamard sequences. It can be seen from these applications that the genetic algorithm is well suited to efficiently design polyphase sequences especially when the number of parameters for the optimization problem is large.
\end{abstract}

\section{INTRODUCTION}

In direct sequence code division multiple access (DSCDMA) systems, sequences in a spreading code should have low cross-correlation (CC) values to suppress multiple access interference (MAI). The auto-correlation (AC) function of the sequences on the other hand, should have a narrow peak to avoid inter-symbol interference (ISI) and to enable proper synchronization. Since good CC comes at the expense of the AC properties, a trade-off between these two performance characteristics has to be accepted and derived by using efficient optimization techniques.

In this paper, we investigate the design of polyphase sequences with low maximum aperiodic cross-correlation and favorable maximum out-of-phase aperiodic auto-correlation [1], [2]. The two classes of polyphase sequences considered are the Oppermann sequences [3], [4], which offer a wide range of correlation properties and the modified WalshHadamard sequences [5], which have been shown to offer good correlation properties. Since the number of parameters for the optimization problem is large, especially for the modified Walsh-Hadamard sequences, it is difficult if not impossible to use global optimization methods for solving such problems. Thus, we propose to transform the problem with continuous variables to another problem with discrete variables. This problem can then be solved efficiently using a genetic algorithm [6], [7]. These types of algorithms have been successfully applied in various areas such as neural networks [8], however, to the authors' best knowledge, their use for the design of polyphase sequences has been rather sparse.

This paper is organized as follows. Section II defines the maximum aperiodic correlation values and the maximum nontrivial aperiodic correlation values which will serve to formulate fitness functions for the genetic algorithm. In Section III, the Oppermann sequences and the modified Walsh-Hadamard sequences are described as well as the formulation of the optimization problem. The genetic algorithm for the efficient design of polyphase sequences is described in Section IV. The subsequent Section $\mathrm{V}$ provides a design example along with some insights into the convergence behavior of the genetic algorithm. Section VI concludes the paper.

\section{CORRELATION MEASURES}

As far as performance analysis for wireless communication systems is concerned, the focus has changed from periodic to aperiodic correlation measures, e.g. [3].

Let $N$ denote the sequence length. The aperiodic correlation $c_{k, l}(i)$ between a pair of sequences $\mathbf{u}_{k}=$ $\left[u_{k}(0), \cdots, u_{k}(N-1)\right]$ and $\mathbf{u}_{l}=\left[u_{l}(0), \cdots, u_{l}(N-1)\right]$ is defined as

$c_{k, l}(i)= \begin{cases}\frac{1}{N} \sum_{v=0}^{N-1-i} u_{k}(v) u_{l}^{*}(v+i), & 0 \leq i \leq N-1 \\ \frac{1}{N} \sum_{v=0}^{N-1+i} u_{k}(v-i) u_{l}^{*}(v), & 1-N \leq i<0 \\ 0, & |i| \geq N\end{cases}$

where '*' denotes the complex conjugate of a complex variable and integer $i$ denotes the shift parameter. Given a set $\mathcal{U}=$ $\left\{\mathbf{u}_{1}, \mathbf{u}_{2}, \cdots, \mathbf{u}_{S}\right\}$ of $S$ sequences, the maximum out-of-phase aperiodic auto-correlation value $c_{\alpha m}(\mathcal{U})$ is defined as

$$
c_{a m}(\mathcal{U})=\max _{\substack{1 \leq k \leq S \\ 1 \leq i \leq N-1}}\left|c_{k, k}(i)\right|
$$

while the maximum aperiodic cross-correlation value $c_{c m}(\mathcal{U})$ is defined as

$$
c_{c m}(\mathcal{U})=\max _{\substack{1 \leq k, l \leq S, k \neq l \\ 0 \leq i \leq N-1}}\left|c_{k, l} l(i)\right|,
$$


respectively. Based on the maximum aperiodic correlation values $c_{a m}(\mathcal{U})$ and $c_{c m}(\mathcal{U})$, the maximum nontrivial aperiodic correlation value $c_{\text {max }}(\mathcal{U})$ is defined as

$$
c_{\text {max }}(\mathcal{U})=\max \left\{c_{a m}(\mathcal{U}), c_{c m}(\mathcal{U})\right\} .
$$

This correlation value will be used to formulate fitness functions for the genetic algorithm. However, it should be noted that the algorithm can be easily applied to incorporate other correlation measures as the fitness function.

\section{Classes of Polyphase Sequences and OPTIMIZATION PROBLEMS}

\section{A. Oppermann Sequences}

A family of complex spreading sequences with a wide range of correlation properties is proposed in [3]. For $1 \leq k \leq N$, the $(v-1)^{t h}$ element $u_{k}^{o}(v-1)$ of an Oppermann sequence $\mathbf{u}_{k}^{o}$ is defined by

$$
u_{k}^{o}(v-1)=(-1)^{k v} \exp \left[\frac{j \pi\left(k^{m} v^{p}+v^{n}\right)}{N}\right], \quad 1 \leq v \leq N,
$$

where $m, n$ and $p$ take real values. For different combinations of $m, n$ and $p$, all the sequences have the same auto-correlation magnitude. This magnitude depends only on $n$ if $p=1$ [3]. In view of this property, we only consider the case of $p=1$. In [4], the design of this type of sequences with respect to the average mean-square aperiodic auto-correlation and average mean-square aperiodic cross-correlation is investigated. In this paper, we consider the problem of minimizing the maximum nontrivial aperiodic correlation value. A genetic algorithm is presented to solve the optimization problem. The optimization problem can be posed as

$$
\min _{m \in\left[m_{1}, m_{2}\right), n \in\left[n_{1}, n_{2}\right)} c_{\max }\left(\mathcal{U}^{o}\right),
$$

where $\left[m_{1}, m_{2}\right)$ and $\left[n_{1}, n_{2}\right)$ are the search regions for $m$ and $n$, respectively, and $\mathcal{U}^{\circ}=\left\{\mathbf{u}_{1}^{o}, \cdots, \mathbf{u}_{N}^{o}\right\}$. The problem (6) is an optimization problem with two continuous variables $m$ and $n$. This problem can be approximated by an integer discrete optimization problem by restricting the continuous parameters $m$ and $n$ within $b$ binary bits, where $b$ is a pre-defined number of bits. These parameters are transformed to discrete variables as follows

$$
m=\left(m_{2}-m_{1}\right) \mathbf{x}_{1}^{T} \mathbf{g}(b)+m_{1}
$$

and

$$
n=\left(n_{2}-n_{1}\right) \mathbf{x}_{2}^{T} \mathbf{g}(b)+n_{1}
$$

where $\mathbf{x}_{1}$ and $\mathbf{x}_{2}$ are $b \times 1$ binary vectors and

$$
\mathbf{g}(b)=\left[2^{-1}, \cdots, 2^{-b}\right]^{T} \text {. }
$$

The grid size of the search region for $m$ and $n$ are $\left(m_{2}-\right.$ $\left.m_{1}\right) / 2^{b}$ and $\left(n_{2}-n_{1}\right) / 2^{b}$, respectively, which are small for large $b$. Thus, the problem (6) can be approximated as the following integer discrete optimization problem:

$$
\left\{\begin{array}{l}
\min _{\mathbf{x}_{1}, \mathbf{x}_{2}} c_{\max }\left(\mathcal{U}^{o}\right) \\
\mathbf{x}_{1}, \mathbf{x}_{2} \text { are } b \times 1 \text { binary vectors. }
\end{array}\right.
$$

\section{B. Modified Walsh-Hadamard Sequences}

The second considered class of sequences is based on the modified Hadamard matrix $\tilde{\mathbf{H}}_{N}$ of size $N \times N$ as suggested in [5], where $N$ is a power-of-two. This modified Hadamard matrix can be obtained from the conventional Hadamard matrix $\mathbf{H}_{N}$ of size $N \times N$ and a diagonal matrix $\mathbf{D}_{N}$ of size $N \times N$ as

$$
\tilde{\mathbf{H}}_{N}=\mathbf{H}_{N} \mathbf{D}_{N}
$$

where the Hadamard matrix can be calculated from the Kronecker product

$$
\mathbf{H}_{N}=\mathbf{H}_{N / 2} \otimes \mathbf{H}_{2} \text {, where } \mathbf{H}_{2}=\left[\begin{array}{ll}
+1 & +1 \\
+1 & -1
\end{array}\right] \text { and } \mathbf{H}_{\mathbf{1}}=[+1]
$$

while the diagonal matrix is given by

$$
\mathbf{D}_{N}=\operatorname{diag}\left\{\left[\exp \left(j 2 \pi d_{1}\right), \cdots, \exp \left(j 2 \pi d_{N}\right)\right]\right\}
$$

and $d_{k} \in[0,1)$, for all $1 \leq k \leq N$. The $N$ modified WalshHadamard sequences of the set $\mathcal{U}^{M}=\left\{\mathbf{u}_{1}^{M}, \mathbf{u}_{2}^{M}, \cdots, \mathbf{u}_{N}^{M}\right\}$ form the rows of the matrix $\tilde{\mathbf{H}}_{N}$.

Similar to the design of the Oppermann sequence, the search for the minimum $c_{\max }\left(\mathcal{U}^{M}\right)$ is restricted to $q$ bits for each parameter $d_{k}$ in the interval $[0,1)$. For $1 \leq k \leq N, d_{k}$ can then be expressed as

$$
d_{k}=\mathbf{z}_{k}^{T} \mathbf{g}(q),
$$

where $\mathbf{z}_{k}$ is a $q \times 1$ binary vector. The design of the modified Walsh-Hadamard sequences with minimum $c_{\max }\left(\mathcal{U}^{M}\right)$ can then be transformed to the following discrete optimization problem

$$
\left\{\begin{array}{l}
\min _{\mathbf{z}_{1}, \cdots, \mathbf{z}_{N}} c_{\max }\left(\mathcal{U}^{M}\right), \\
\mathbf{z}_{k} \text { are } q \times 1 \text { binary vectors for all } 1 \leq k \leq N .
\end{array}\right.
$$

The number of parameters for the problem (14) is $q N$, which is large and can increase quickly with $q$ and $N$. Hence, it is difficult if not impossible to use global optimization methods for solving such problems whereas the genetic algorithm as specified in the next section turns out to be well suited to solve this type of problems.

\section{GENETIC ALGORITHM}

The problems (10) and (14) are integer discrete optimization problems with binary variables. These problems can be solved efficiently by using the genetic algorithm [6], [7] which is a stochastic search method that mimics the metaphor of natural biological evolution. The advantage of the method is its significant computational saving over other global discrete optimization methods.

The genetic algorithm can be described as follows. Initially, the algorithm requires an initial set of $I$ members with each member defined as a chromosome. This initial set is randomly generated and referred to as a population. For the Oppermann sequences, each member of the population is a $2 b$ binary sequence $\left[\mathbf{x}_{1}, \mathbf{x}_{2}\right]$. Similarly, for the modified Walsh-Hadamard sequences, each member is a $q N$ binary sequence $\left[\mathbf{z}_{1}, \cdots, \mathbf{z}_{N}\right]$ : Let us denote the families of spreading 
sequences corresponding to the above two populations as $\left\{\mathcal{U}_{1}^{o}, \cdots, \mathcal{U}_{I}^{o}\right\}$ and $\left\{\mathcal{U}_{1}^{M}, \cdots, \mathcal{U}_{I}^{M}\right\}$, respectively. The cost function for each member in the population is evaluated and a corresponding fitness value is then assigned. In the context of polyphase sequence design, the fitness function for the problems (10) and (14) can be defined, respectively, as

$$
f_{i}^{o}=\frac{I / c_{\max }\left(\mathcal{U}_{i}^{o}\right)}{\sum_{k=1}^{I} 1 / c_{\max }\left(\mathcal{U}_{k}^{o}\right)}, 1 \leq i \leq I
$$

and

$$
f_{i}^{M}=\frac{I / c_{\max }\left(\mathcal{U}_{i}^{M}\right)}{\sum_{k=1}^{I} 1 / c_{\max }\left(\mathcal{U}_{k}^{M}\right)}, 1 \leq i \leq I .
$$

Obviously, it follows from (15) and (16) that the fitness value corresponding to a set or family $\mathcal{U}$ of sequences with low cost function $c_{\max }(\mathcal{U})$ is high and vice versa. Moreover, we have the following condition on the fitness function

$$
0 \leq f_{i}^{o} \leq I, \forall i \text { and } \sum_{i=1}^{I} f_{i}^{o}=I
$$

and

$$
0 \leq f_{i}^{M} \leq I, \forall i \text { and } \sum_{i=1}^{I} f_{i}^{M}=I .
$$

The second step is to form an intermediate population. In this case, the stochastic universal sampling [9] is chosen for the selection process. For each chromosome, the integer portion of the fitness function indicates the number of copies of that chromosome that are directly placed in the intermediate population. The fractional portion of the fitness function indicates the probability of placing that chromosome in the intermediate population. After the selection process has been carried out, a new population is obtained by combining the intermediate population with the current population.

The third step is to perform the crossover and the mutation operations. Crossover is applied to random pair sequences with a probability $p_{c}$. The two types of crossover: (1) 1point crossover and (2) random crossover are selected at random. After the crossover, the mutation operation is applied. Mutation is the phenomenon where a random " 0 " becomes "l" and a " 1 " becomes " 0 " with a small probability $p_{m}$. Assume that this operation gives rise to $I_{c}$ new members with a new population having $I+I_{c}$ members. The fitness values (15) and (16) for all the members of this new population are obtained. The process of natural selection is used to reduce this population to $I$ members. These $I$ members are selected as those with the highest fitness values. The above process is repeated and the algorithm is terminated if either the variance of the fitness function for the $I$ members in the population falls below a selected threshold, or the number of iterations is greater than a defined value.

A summary of the genetic algorithm used for the design of polyphase sequences is given as follows:
Algorithm 4.1: Genetic algorithm

- Step 1: Select the initial population of $I$ members randomly. Calculate the fitness function of all members in the population according to (15) and (16), respectively.

- Step 2: Obtain the intermediate population by using the stochastic universal sampling method.

- Step 3: Perform crossover and mutation on the intermediate population to obtain a new population with $I+I_{c}$ members. Apply the natural selection process to reduce this population to $I$ members.

- Step 4: Repeat the Steps 2-3. Stop the procedure if the variance of the fitness function in the population falls below a threshold or the number of iterations is greater than a defined value.

\section{DESIGN EXAMPLES}

Consider the design of the Oppermann sequences and the modified Walsh-Hadamard sequences of length $N=32$ using the genetic algorithm. The initial size of the population is chosen as $I=400$ and $I_{c}$ is chosen as $I_{c}=I$.

The search for the Oppermann sequences is performed in the intervals $\left[m_{1}, m_{2}\right)=[0,4)$ and $\left[n_{1}, n_{2}\right]=[0,4)$ with the number of bits $b=14$. Thus, the number of binary variables for the optimization problem $(10)$ is $14 \times 2=28$.

The search for the modified Walsh-Hadamard sequences is performed with the number of bits $q$ increasing from 1 to 4 . The number of binary variables for these cases are 32,64 , 96 and 128, respectively. For both problems, the crossover probability $p_{c}$ is chosen close to one while the mutation probability $p_{m}$ is chosen as a small value, $p_{m}=0.01$.

Table 1 shows the optimum values for the design example of spreading sequences. The first row of the table is the result for the Oppermann sequences while the rest of the table are the results for the modified Walsh-Hadamard sequences. It can be seen from the table that the Oppermann sequences in this example perform superior compared to the modified Walsh-Hadamard sequences and offers the lowest maximum nontrivial correlation value. For the modified Walsh-Hadamard sequences, the value of $c_{\max }\left(\mathcal{U}^{M}\right)$ improves slightly with an increase in the number of bits $q$.

Fig. 1 shows convergence of the genetic algorithm for the mean values of the cost function for the Oppermann sequences, given by

$$
\frac{1}{I} \sum_{i=1}^{I} c_{\max }\left(\mathcal{U}_{i}^{o}\right)
$$

and is depicted for different iterations. The algorithm converges relatively fast, within $15-20$ iterations. The figure also shows auto-correlation magnitude and frequency spectra for the first three sequences taken from the spreading code with lowest $c_{\max }\left(\mathcal{U}^{\circ}\right)$. It can be seen from the figure that the autocorrelation has a distinct pick while the frequency spectra are distinct for high power levels.

Fig. 2 shows the convergence for the mean value of the cost function for the modified Walsh-Hadamard sequences for the last case with $b=4$. The algorithm converges slower 
TABLE I

OPTIMUM $c_{\max }(\mathcal{U})$ FOR THE OPPERMANN SEQUENCES AND THE MODIFIED WALSH-HADAMARD SEQUENCES.

\begin{tabular}{|c|c|c|c|c|c|}
\hline Sequences & Number of bits & Number of variables & $c_{\max }(\mathcal{U})$ & $c_{a m}(\mathcal{U})$ & $c_{c m}(\mathcal{U})$ \\
\hline Oppermann sequences & $b=14$ & 28 & 0.2776 & 0.2308 & 0.2776 \\
\hline \multirow{3}{*}{ Modified Hadamard sequences } & $q=1$ & 32 & 0.4063 & 0.3438 & 0.4063 \\
\cline { 2 - 7 } & $q=2$ & 64 & 0.3494 & 0.3263 & 0.3494 \\
\cline { 2 - 7 } & $q=3$ & 96 & 0.3463 & 0.3330 & 0.3463 \\
\cline { 2 - 7 } & $q=4$ & 128 & 0.3439 & 0.3377 & 0.3439 \\
\hline
\end{tabular}

than with the Oppermann sequence since the number of binary variables increased to 128 . The number of search combinations in this case is approximately $400 \times 55=22000$, which is significantly smaller than the total number of combinations $2^{128}=3.4028 \times 10^{38}$. The figure also shows auto-correlation magnitude and frequency spectra for the first three sequences taken from the spreading code with lowest $c_{\text {max }}\left(\mathcal{U}^{M}\right)$. The first two sequences have the same auto-correlation magnitude while the third one has a different magnitude.

\section{CONCLUSIONS}

In this paper, a genetic algorithm is proposed for designing polyphase sequences with optimized correlation characteristics. The maximum nontrivial aperiodic correlation values are used to specify the cost or fitness function of the optimization problems. Since the number of parameters for the optimization problem can be large, it is difficult if not impossible to use global optimization methods for solving such problems whereas the genetic algorithm can cope very well with these type of scenarios. The genetic algorithm is applied to the design of Oppermann sequences and modified Walsh-Hadamard sequences. It can be seen from these design examples that the genetic algorithm is well suited to efficiently design polyphase sequences especially when the number of parameters for the optimization problem is large.

\section{ACKNOWLEDGEMENT}

This work was partly supported by the Commonwealth of Australia through the Cooperative Research Centre program.

\section{REFERENCES}

[1] D. V. Sarwarte and M. B. Pursley, "Crosscorrelation Properties of Pseudorandom and Related Sequences," Proc. of the IEEE, vol. 68, no. 5, May 1980, pp. 593-619, 1980.

[2] P. Fan and M. Damell, "Sequence Design for Communications Applications." New York, John Wiley \& Sons, 1996.

[3] 1. Oppermann and B. S. Vucetic, "Complex Spreading Sequences with a Wide Range of Correlation Properties," IEEE Trans. on Commun., vol. 45 , pp. $365-375,1997$.

[4] H. H. Dam, H.-J Zepernick, S. Nordholm, and J. Nordberg, "Design of Spreading Sequences Using a Modified Bridging Method," IEEE Global Commun. Conf., Taipei, Taiwan, vol. 1, pp. $835-840$, Nov. 2002.

[5] B. J. Wysocki and T. A. Wysocki, "Modified Walsh-Hadamard Sequences for DS CDMA Wireless Systems," Int. J. Adapt. Control Signal Process., vol. 16, pp. 589-602, 2002.

[6] N. Ansari and E. Hou, "Computational Intelligence for Optimization," Dordrecht, Kluwer Academic Publishers, 1997.

[7] D. E. Goldberg, "Genetic Algorithm in Search Optimization, and Machine Leaming," Reading, Addison-Wesley, 1989.

[8] L. Guo, D.-S. Huang. and W. Zhao, "The Optimization of Radial Basis Probabilistic Neural Networks Based on Genetic Algorithms," in Proc. Int. Joint Conf. on Neural Networks, vol. 4, pp. 3213-3217, Jul. 2003.
[9] J. Baker, "Reducing Bias and Inefficiency in the Selection Algorithm, Proc. of the 2nd Intl. Conf. on Genetic Algorithms, pp.14-21, Jul. 1987.
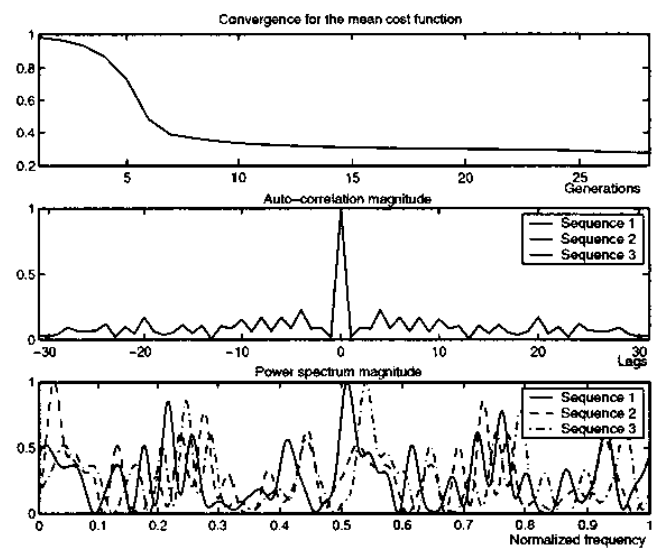

Fig. 1. Convergence and correlation plots for the Oppermann sequences with lowest $c_{\max }\left(\mathcal{U}^{o}\right)$.
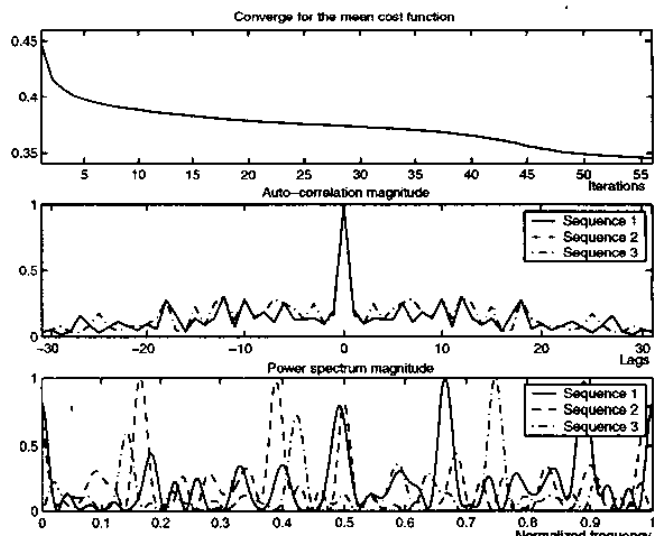

Fig. 2. Convergence and correlation plots for the modified Walsh-Hadamard sequences with lowest $c_{\max }\left(\mathcal{U}^{M I}\right)$. 